\title{
Improvement of left ventricular function by lifestyle intervention in obesity: contributions of weight loss and reduced insulin resistance
}

\author{
W. Kosmala • T. O'Moore-Sullivan • R. Plaksej • \\ M. Przewlocka-Kosmala • T. H. Marwick
}

Received: 15 June 2009 /Accepted: 17 July 2009/Published online: 2 September 2009

(C) Springer-Verlag 2009

\begin{abstract}
Aims/hypothesis Weight excess and insulin resistance mediate the link between obesity and left ventricular dysfunction. We investigated the effect and mechanisms of lifestyle modification on left ventricular function changes in obese patients.

Methods Reduction of body weight and insulin resistance was sought using a behavioural intervention programme including dietary restrictions and exercise training in 261 patients (age $45 \pm 13$ years) with BMI $\geq 30 \mathrm{~kg} / \mathrm{m}^{2}$, no history of cardiac disease and a normal stress echocardiogram. Each patient underwent echocardiographic measurement of myocardial deformation and velocity at baseline and at 6 month follow-up.

Results Improvements in left ventricular systolic and diastolic function were demonstrated only in patients with significant reduction of weight and/or insulin resistance. Left ventricular improvement was less frequent in patients
\end{abstract}

W. Kosmala $\cdot$ R. Plaksej · M. Przewlocka-Kosmala

Department of Cardiology, Wroclaw Medical University,

Wroclaw, Poland

T. O'Moore-Sullivan • T. H. Marwick ( $\square)$

University of Queensland Department of Medicine,

Princess Alexandra Hospital,

Ipswich Road, Brisbane Q4102, Australia

e-mail: t.marwick@uq.edu.au

T. O’Moore-Sullivan

Diamantina Institute for Cancer, Immunology and Metabolic Medicine, University of Queensland,

Brisbane, QLD, Australia

T. O'Moore-Sullivan

Department of Diabetes and Endocrinology,

Princess Alexandra Hospital,

Brisbane, QLD, Australia with diabetes than in those without (52\% vs $82 \%$ for strain, $50 \%$ vs $81 \%$ for strain rate and $59 \%$ vs $80 \%$ for peak early diastolic myocardial velocity). The independent predictors of improved left ventricular systolic function (increase in strain) were: weight reduction $(\beta=0.14, p<0.05)$, decrease in the HOMA insulin resistance index $(\beta=0.20, p<0.005)$ and absence of diabetes $(\beta=0.18, p<0.02)$. A decrease in $\mathrm{HbA}_{1 \mathrm{c}}$ also predicted improvement of left ventricular diastolic function $(\beta=0.26, p<0.001)$. There was a parallel increment in exercise capacity with intervention and increase in strain was independently correlated with increase in $\dot{V} \mathrm{O}_{2}(\beta=0.13, p<0.04)$.

Conclusions/interpretation Effective lifestyle modifications in obese patients improve left ventricular systolic and diastolic function, but appear less effective with coexisting diabetes. The reversal of left ventricular function abnormalities is associated with reduction of both weight and insulin resistance, and is accompanied by an increase in cardiorespiratory fitness.

Keywords Echocardiography - Insulin resistance .

Lifestyle intervention · Myocardial dysfunction · Obesity

Abbreviations
$\begin{array}{ll}\text { Em } & \text { Peak early diastolic velocity } \\ \text { HOMA-IR } & \text { HOMA index of insulin resistance } \\ \text { SR } & \text { Peak systolic strain rate } \\ \dot{V} \mathrm{O}_{2} & \text { Peak oxygen consumption }\end{array}$

\section{Introduction}

Obesity is now a worldwide epidemic [1], with more than one of three US American adults ranked as obese [2]. The 
public health ramifications are considerable, as obesity is an established risk factor for the development of heart failure, with effects on cardiovascular morbidity and mortality rates independent of commonly associated co-morbidities, such as coronary artery disease, hypertension and diabetes mellitus [3]. Subclinical left ventricular dysfunction is a common finding in overweight patients $[4,5]$ and may progress over time to overt heart disease. As weight excess and insulin resistance are considered to mediate the link between obesity and cardiac function $[5,6]$, successful strategies for weight reduction and improvement in metabolic status could improve left ventricular performance. However, diabetes imposes an additional metabolic burden on the heart, which may modulate the effects of weight loss on left ventricular function.

The most effective weight-reducing approach is gastric bypass surgery, but this is reserved for selected, usually morbidly obese patients [7-10]. Lifestyle modifications (energy intake restriction and increased physical activity) are essential in the management of obesity and its consequences, with even modest weight reduction (5-10\%) likely to promote favourable alterations in cardiometabolic risk $[11,12]$. However, the benefits of these steps on left ventricular function are less well defined. Therefore, by following myocardial deformation to detect subclinical left ventricular dysfunction in overweight participants [5, 13], we sought: (1) to investigate the effect of a lifestyle intervention with reduced dietary intake and exercise training on left ventricular performance; and (2) to identify the factors associated with a beneficial effect on cardiac function.

\section{Methods}

\section{Patient selection}

We prospectively evaluated 261 obese men and women $\left(\mathrm{BMI} \geq 30 \mathrm{~kg} / \mathrm{m}^{2}\right.$ ) with cardiovascular risk factors, who were referred to university hospitals from general practice and specialist clinics for management of obesity. All participants underwent a medical evaluation comprising clinical history, routine blood analysis, lipid profile, a 75 g OGTT (in patients without known diabetes), electrocardiography and echocardiography. Patients with a history of CHD were excluded, as were those with abnormal stress electrocardiograms or echocardiograms, or with non-diagnostic stress tests to $<85 \%$ predicted maximum heart rate. Other exclusion criteria included: any known heart disease (including a history or findings suggesting heart failure), thyroid and renal diseases, pregnancy and chronic alcohol consumption.

Diabetes mellitus was diagnosed according to the criteria recommended by the American Diabetes Association [14]. Patients on oral hypoglycaemic therapy were eligible for recruitment, but those being treated with insulin were excluded. Hypertension was defined by antihypertensive treatment or documented blood pressure measurements $\geq 140 \mathrm{mmHg}$ systolic and/or $\geq 90 \mathrm{mmHg}$ diastolic on at least two occasions. The metabolic syndrome was recognised as recommended by the International Diabetes Federation [15]. Cardiovascular risk was calculated according to the Framingham risk score [16]. Among cardiovascular risk factors considered were age, sex, total cholesterol, HDL-cholesterol, blood pressure, smoking status and presence of diabetes.

All participants were informed of the purpose of the study and provided written informed consent. Investigations were in accordance with the Declaration of Helsinki as revised in 2000 and were approved by the institutional ethics committees.

\section{Description of intervention}

Participants attended the lifestyle intervention programme, which included dietary restrictions and increased physical activity for 6 months and was targeted to achieve an energy deficit of 14.6-29.3 MJ/week. Exercise training encompassed an individualised exercise programme with at least 30 min of moderate-intensity physical activity on most days (a minimum approximately $150 \mathrm{~min}$ per week) as recommended by the American College of Sports Medicine and the International Association for the Study of Obesity [17, 18]. This exercise regimen is believed to lead to burning of fat stores, which occurs after the first $20 \mathrm{~min}$ of exercise with preferential utilisation of glycogen. Dietary modification consisted of personal advice by a dietitian with a view to reducing daily energy intake to 3.3-6.3 MJ and with $<30 \%$ of energy requirement provided by lipids. We sought to avoid changes to medication during the observation.

The study sample was divided into two groups according to compliance with lifestyle modifications: (1) participants with good adherence demonstrating an arbitrary weight loss $>5 \%$ at the end of the intervention programme; and (2) those with poor adherence and unable to achieve a $5 \%$ decrease in body weight.

\section{Clinical evaluation}

Anthropometric assessment including height, body weight and both hip and waist circumference was carried out after an overnight fast. Brachial blood pressure was estimated using standard sphygmomanometry with the participant in the sitting position. Measurements were repeated three times after at least 5 min of rest and then averaged.

\section{Biochemical assays}

Blood samples were obtained between 08:00 and 09:00 hours after overnight fast and supine rest for $30 \mathrm{~min}$, and before 
taking hypoglycaemic and antihypertensive drugs, exercise testing or echocardiography. Serum glucose was measured by enzymatic assay (Dade Behring, Newark, DE, USA). Serum insulin was evaluated using a chemiluminescent enzyme immunoassay (Immulite 2000; Diagnostic Products, Los Angeles, CA, USA). The HOMA index of insulin resistance (HOMA-IR) was computed as previously reported [19]. A $75 \mathrm{~g}$ OGTT was performed in each participant without prior diagnosis of diabetes.

\section{Body composition}

Body fat percentage was evaluated by dual energy X-ray absorptiometry (Discovery-W; Hologic, Bedford, MA, USA).

Metabolic exercise testing

Exercise testing was carried out on a treadmill exerciser according to a protocol individualised to the exercise capacity of the patients. Measurements of peak oxygen consumption $\left(\dot{V} \mathrm{O}_{2}\right)$ were performed using breath-by-breath analyses of expired gas (V29C; Sensormedics, Yorba Linda, CA, USA; and QPLEX; Quinton Instruments, Seattle, WA, USA).

\section{Echocardiography}

Echocardiographic examinations were performed using standard systems (Vivid 7 and System Five; GE Medical Systems, Horten, Norway) equipped with phased array 2.5 MHz multifrequency transducers.

Conventional echocardiography Left ventricular and left atrial dimensions and wall thicknesses were measured according to standard recommendations [20]. Left ventricular mass was determined from the modified American Society of Echocardiography cube formula and normalised for height to the power of 2.7 to achieve the left ventricular mass index [21]. A modified Simpson's biplane method was used to calculate left ventricular ejection fraction.

Pulsed wave Doppler recordings of the left ventricular inflow were obtained from the apical four-chamber view with the sample volume placed between the tips of the mitral leaflets. The peak early and late diastolic flow velocities, the ratio of peak early and late diastolic flow velocities, and the deceleration time of early diastolic flow were evaluated.

Tissue Doppler imaging To assess left ventricular longitudinal function, real-time colour tissue Doppler myocardial imaging data were acquired in the three apical views. The narrowest possible image sector angle and the optimal depth of imaging were used to achieve the maximal frame rate. Pulse repetition frequency was adjusted at the lowest level without aliasing. The sampled region was oriented as parallel as possible to the myocardial segment of interest to ensure the imaging angle did not exceed $20^{\circ}$. The region of interest was continuously positioned by manual tracking in the mid-myocardial layer of each segment during the heart cycle. Digital data were stored in a cine-loop format, transferred to a dedicated workstation (Echopac; GE Medical Systems) and subsequently analysed off-line.

Myocardial velocities Regional myocardial velocity curves were extracted from colour Doppler data obtained in the basal segments of the interventricular septum in order to assess peak systolic velocity and peak early diastolic velocity (Em). Pulsed-wave tissue Doppler was recorded in the septal mitral annulus. The ratio of mitral inflow early diastolic velocity to the mitral annular Em was computed as an approximation of left ventricular filling pressure [22].

Myocardial deformation Left ventricular myocardial deformation curves were derived from the apical, mid and basal segments of each left ventricular wall. Strain-rate was evaluated from the tissue velocity gradient over a computation length of $12 \mathrm{~mm}$. Strain rate profiles were then integrated over time to obtain the natural strain curves using end-diastole defined to occur at the R-peak of ECG as the reference point. The analysis of left ventricular deformation curves included peak strain, identified as the greatest negative value on the strain curve, and peak systolic strain rate (SR). All strain, strain rate and myocardial velocity profiles were averaged over three consecutive heart cycles and patient results were expressed as the average values from all segments.

All echocardiograms were obtained and analysed by readers blinded to patients' clinical data.

Feasibility No participants were excluded due to poor imaging quality. Despite great care during acquisition, $7 \%$ of all left ventricular segments were disqualified from further analysis due to unreliable interpretation of myocardial velocity and deformation profiles resulting from artefacts, poor signal:noise ratio or unfavourable angle of insonation. The reproducibility of tissue Doppler measurements was estimated in 15 randomly selected participants. The intra- and inter-observer variability was $1.6 \pm 1.2 \%$ and $1.8 \pm 1.2 \%$ for strain, $0.1 \pm 0.1$ and $0.1 \pm 0.1 \mathrm{~s}^{-1}$ for SR, and $0.2 \pm 0.8$ and $0.3 \pm 0.7 \mathrm{~cm} / \mathrm{s}$ for myocardial velocities, respectively.

Statistical analysis

Data are presented as mean $\pm \mathrm{SD}$; skewed variables were logtransformed. Intergroup comparisons were performed by an unpaired two-sided Student's $t$ test or, with more than two groups included, by one-way ANOVA with Scheffe's post hoc test for continuous variables and by $\chi^{2}$ for categorical 
variables. Homogeneity of variances was evaluated by the Levene test. Longitudinal analyses were carried out using a repeated-measures two-way ANOVA with planned comparisons by the linear contrasts method. Associations between variables were studied using Pearson's correlation coefficient and stepwise multiple regression analysis. A receiver operator characteristic analysis was used to examine the ability of particular variables to predict left ventricular function improvement. Differences in the area under the receiver operator characteristic curves were analysed using the $Z$ test [23]. Improvement of left ventricular function was defined in analyses by an increase in each variable (strain, SR and Em). Calculations were carried out with standard statistical software (Statistica for Windows 7; StatSoft, Tulsa, OK, USA). A value of $p<0.05$ was accepted as statistically significant.

\section{Results}

Patient characteristics

The baseline characteristics are representative of persons enrolling in our weight loss programmes, i.e. middle-aged $(45 \pm 13$ years) and predominantly female sex (64\%). Postmenopausal status was established in 50 of the women participants (30\%). Hypertension (36\%), metabolic syndrome (62\%) and type 2 diabetes mellitus (59\%) were frequent, as were treatments for hypertension (ACE inhibitors/angiotensin receptor blockers $30 \%$, beta-blockers $4 \%$, calcium channel antagonists $12 \%$, diuretics 29\%) and diabetes (biguanides $63 \%$, sulfonylureas $26 \%$, no thiazolidinediones). The duration of follow-up was similar in the adherent and non-adherent groups ( $6.0 \pm 1.7$ vs $6.1 \pm 1.6$ months, $p=0.81$ ).

The adherent patients were younger, more commonly women and less likely to have diabetes, and had lower baseline insulin, glucose, HOMA-IR, $\mathrm{HbA}_{1 \mathrm{c}}$, waist circumference and WHR, and higher exercise capacity and Em than their peers from the poor adherence group (Tables 1 and 2). The 10-year risk of coronary heart disease (assessed by Framingham scoring) was $1 \%$ in women and $6 \%$ in men, and similar in both groups.

\section{Effect of the lifestyle modifications}

Participants with good adherence showed a significant decrease in weight and adiposity. They also experienced improvements in insulin resistance, lipid profile, cardiorespiratory fitness and left ventricular systolic and diastolic function, as well as reductions of left ventricular mass index at the end of the follow-up. The intervention caused no changes in clinical, laboratory and echocardiographic variables in the non-adherent group (Tables 1 and 2). Table 3 compares the changes in metabolic and imaging variables in groups with baseline diabetes mellitus, hypertension or both. There was no difference in any variable between hypertension and non-hypertension patients, but important differences were seen between groups with or without diabetes.

Categorical improvements in strain, SR and Em with the lifestyle intervention were demonstrated in $82 \%, 81 \%$ and $80 \%$ of patients without diabetes respectively, compared with $52 \%, 50 \%$ and $59 \%$ of patients with diabetes, respectively $(p<$ 0.001 for all).

Correlates of alterations in left ventricular function

The correlates of changes in left ventricular function following the lifestyle modifications in patients who adhered to the intervention are presented in Table 4 . Changes in systolic function were independently associated with baseline systolic performance and metabolic alterations ( $\triangle$ HOMA-IR, $\triangle$ BMI, co-existence of diabetes); diastolic changes were independently associated with baseline diastolic performance and metabolic alterations ( $\triangle \mathrm{BMI}$, $\left.\Delta \mathrm{HbA}_{1 \mathrm{c}}\right)$. Comparison of the AUCs in the prediction of left ventricular function improvement revealed equivalent values of $\triangle$ HOMA-IR and $\triangle \mathrm{BMI}$ for systolic and diastolic variables (Fig. 1a-f). Among other factors, only $\Delta \mathrm{HbA}_{1 \mathrm{c}}$ emerged as a significant predictor of left ventricular diastolic improvement $(\triangle \mathrm{Em})(\mathrm{AUC}=0.69, \mathrm{SE}=0.058, p<0.001)$.

Change in strain was an independent correlate of $\Delta \dot{V} \mathrm{O}_{2}$ even after adjustment for other variables including age, presence of diabetes, changes in BMI, HOMA-IR, left ventricular mass index, blood pressure and pharmacotherapy $(\beta=0.13, p<0.04)$.

Influence of weight loss and insulin resistance reduction on left ventricular function and cardiorespiratory fitness improvement

The study population was re-classified on the basis of predefined cut-points of 5\% decrease in body weight (used for the initial identification of adherence to the intervention) and $20 \%$ decrease in HOMA-IR to create the following four groups: (1) improvement in body weight and insulin resistance (BMI+IR+, $n=79)$; (2) improvement in body weight alone (BMI+IR-, $n=45)$; (3) improvement in insulin resistance alone (BMI-IR+, $n=43$ ); and (4) no improvement in either (BMI-IR-, $n=94)$. Average weight change ranged from a reduction of $14 \pm 8 \mathrm{~kg}$ in the $\mathrm{BMI}+\mathrm{IR}+$ group to an increase of $2 \pm 10 \mathrm{~kg}$ in the BMI-IR- group. HOMA-IR change varied from $43 \%$ less to $41 \%$ more.

Each group with improvement in at least one of the two variables (i.e. $\mathrm{BMI}+\mathrm{IR}+$, $\mathrm{BMI}-\mathrm{IR}+$, $\mathrm{BMI}+\mathrm{IR}-$ ) demonstrated improvements in left ventricular systolic $(\Delta$ strain $)$ and diastolic $(\Delta \mathrm{Em})$ functions. The cumulative effect of 
Table 1 Demographic data, and baseline and follow-up clinical characteristics of the study group

\begin{tabular}{|c|c|c|c|c|c|c|c|c|}
\hline \multirow[t]{2}{*}{ Variables } & \multicolumn{3}{|c|}{ Adherent $(n=124)$} & \multicolumn{3}{|c|}{ Non-adherent $(n=137)$} & \multicolumn{2}{|c|}{$p$ value, adherent vs non-adheren } \\
\hline & Baseline & Follow-up & $p$ value & Baseline & Follow-up & $p$ value & Baseline & Follow-up \\
\hline \multicolumn{9}{|c|}{ Demographic and clinical features } \\
\hline Age (years) & $41 \pm 13$ & - & & $49 \pm 12$ & - & & 0.0001 & - \\
\hline Sex $(\%$ male $)$ & 24 & - & & 47 & - & & 0.001 & - \\
\hline Diabetes $(\%)$ & 36 & - & & 80 & - & & 0.0001 & - \\
\hline Hypertension (\%) & 35 & & & 36 & & & 0.81 & \\
\hline Smoking $(\%)$ & 17 & & & 25 & & & 0.13 & \\
\hline Body weight (kg) & $102 \pm 18$ & $90 \pm 17$ & 0.0001 & $100 \pm 19$ & $101 \pm 20$ & 0.06 & 0.42 & 0.0001 \\
\hline $\operatorname{BMI}\left(\mathrm{kg} / \mathrm{m}^{2}\right)$ & $36.4 \pm 5.7$ & $31.2 \pm 5.4$ & 0.0001 & $35.1 \pm 5.5$ & $35.5 \pm 6.0$ & 0.07 & 0.07 & 0.0001 \\
\hline Body fat $(\%)$ & $39.5 \pm 10.1$ & $37.3 \pm 9.5$ & 0.0001 & $39.0 \pm 8.2$ & $39.4 \pm 8.7$ & 0.24 & 0.71 & 0.23 \\
\hline Waist circumference $(\mathrm{cm})$ & $107 \pm 12$ & $101 \pm 10$ & 0.0001 & $111 \pm 14$ & $112 \pm 14$ & 0.10 & 0.04 & 0.0001 \\
\hline WHR & $0.92 \pm 0.09$ & $0.89 \pm 0.09$ & 0.01 & $0.95 \pm 0.10$ & $0.94 \pm 0.10$ & 0.27 & 0.03 & 0.0001 \\
\hline Systolic BP (mmHg) & $127 \pm 13$ & $127 \pm 17$ & 0.82 & $129 \pm 15$ & $130 \pm 15$ & 0.4 & 0.35 & 0.38 \\
\hline Diastolic BP (mmHg) & $81 \pm 9$ & $80 \pm 9$ & 0.11 & $81 \pm 9$ & $81 \pm 8$ & 0.64 & 0.71 & 0.43 \\
\hline \multicolumn{9}{|l|}{ Laboratory findings } \\
\hline Fasting insulin (pmol/l) & $103 \pm 48$ & $79 \pm 57$ & 0.0001 & $126 \pm 99$ & $121 \pm 86$ & 0.60 & 0.02 & 0.0001 \\
\hline Fasting glucose $(\mathrm{mmol} / \mathrm{l})$ & $6.0 \pm 2.1$ & $6.0 \pm 2.7$ & 0.69 & $7.8 \pm 3.0$ & $8.2 \pm 3.3$ & 0.10 & 0.0001 & 0.0001 \\
\hline HOMA-IR & $4.1 \pm 2.7$ & $3.4 \pm 2.8$ & 0.04 & $6.1 \pm 5.0$ & $6.4 \pm 5.0$ & 0.45 & 0.0001 & 0.0001 \\
\hline $\mathrm{HbA}_{1 \mathrm{c}}(\%)$ & $6.5 \pm 1.3$ & $6.5 \pm 1.4$ & 0.53 & $7.4 \pm 1.6$ & $7.5 \pm 1.4$ & 0.60 & 0.0001 & 0.0001 \\
\hline LDL (mmol/1) & $3.0 \pm 0.9$ & $2.8 \pm 1.0$ & 0.03 & $2.9 \pm 0.9$ & $2.9 \pm 1.0$ & 0.77 & 0.12 & 0.62 \\
\hline $\mathrm{HDL}(\mathrm{mmol} / \mathrm{l})$ & $1.3 \pm 0.3$ & $1.4 \pm 0.4$ & 0.001 & $1.3 \pm 0.3$ & $1.3 \pm 0.4$ & 0.14 & 0.77 & 0.52 \\
\hline Triacylglycerol (mmol/l) & $1.7 \pm 1.0$ & $1.8 \pm 2.9$ & 0.66 & $2.0 \pm 2.2$ & $2.1 \pm 2.0$ & 0.40 & 0.10 & 0.49 \\
\hline \multicolumn{9}{|l|}{ Exercise capacity } \\
\hline$\dot{V} \mathrm{O}_{2}\left(\mathrm{ml} \mathrm{kg}{ }^{-1} \min ^{-1}\right)$ & $24.8 \pm 9.0$ & $27.1 \pm 9.7$ & 0.001 & $22.4 \pm 7.3$ & $22.8 \pm 7.9$ & 0.19 & 0.03 & 0.001 \\
\hline
\end{tabular}

Unless otherwise indicated values are mean \pm SD

reduced weight and insulin resistance exceeded that of a change in only one variable, evidenced by statistical differences in $\Delta$ strain and $\Delta$ Em between the BMI+IR+ group and the BMI-IR+ or BMI+IR- group.

The enhancement of left ventricular performance seen in patients with BMI and HOMA-IR improvements was accompanied by an increase in peak ventilatory exercise capacity, which was significantly higher in patients from the $\mathrm{BMI}+\mathrm{IR}+$ and $\mathrm{BMI}+\mathrm{IR}-$ groups as compared with the BMI-IR- group (Fig. 2a-e).

Cardiac response to lifestyle modification

Based on changes in body weight, insulin resistance and left ventricular function, we identified two extremes in the cardiac response to lifestyle modification. Cardiac responders included participants from the BMI $+\mathrm{IR}+, \mathrm{BMI}+\mathrm{IR}-$ and $\mathrm{BMI}-\mathrm{IR}+$ groups (with weight reduction $>5 \%$ and/or HOMA-IR decrease $>20 \%$ ), who exhibited improvement in strain. Non-responders were individuals from the BMI-IRgroup and showed no improvement in strain. Cardiac responders were characterised by younger age, lower preva- lence of diabetes and male sex, and lower baseline WHR, strain and SR than non-responders (Table 5).

\section{Discussion}

The main clinical findings of the present study are that improved left ventricular systolic and diastolic function in obese patients is observed in connection with effective lifestyle modifications (i.e. those associated with weight loss and reduced insulin resistance) and less likely in patients with co-existing diabetes. The possibility of left ventricular function improvements during lifestyle intervention is higher in participants who are younger, of female sex, without diabetes and have less extensive visceral fat deposition and more severe baseline cardiac function impairment.

Mechanisms of cardiac function improvement during lifestyle intervention

Lifestyle intervention may improve cardiac structural and functional pathologies through a variety of mechanisms [4-6, 


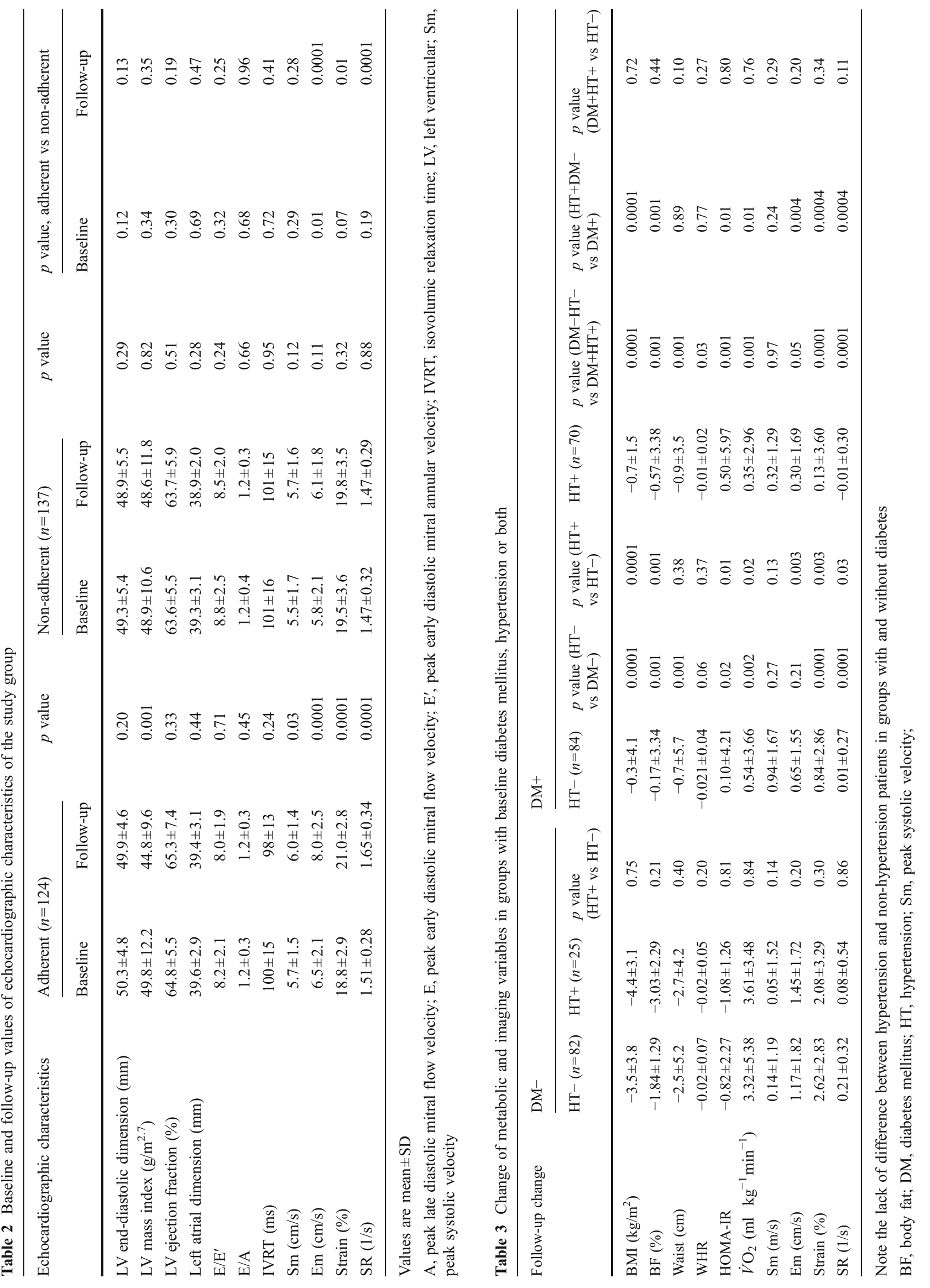


Table 4 Significant independent correlates of changes in left ventricular systolic and diastolic function indices in obese patients with good adherence to the intervention

\begin{tabular}{lccl}
\hline Variables & $R^{2}$ & $\beta$ & $p$ value \\
\hline$\Delta$ Strain & 0.47 & -0.50 & 0.0001 \\
Baseline strain & & 0.20 & 0.005 \\
$\Delta$ HOMA & & -0.18 & 0.02 \\
Diabetes & & 0.14 & 0.05 \\
$\Delta$ BMI & & 0.10 & 0.15 \\
$\Delta$ LVMI & 0.51 & & \\
$\Delta$ SR & & -0.61 & 0.0001 \\
Baseline SR & & -0.47 & 0.0001 \\
Diabetes & & 0.14 & 0.04 \\
$\Delta$ BMI $^{\text {HOMA }}$ & & 0.18 & 0.01 \\
$\Delta$ Em & 0.32 & -0.52 & 0.0001 \\
Baseline Em & & 0.27 & 0.001 \\
$\Delta$ BMI $_{\Delta \text { HbA }}$ & & 0.26 & 0.001 \\
Diabetes & & -0.11 & 0.21 \\
\hline
\end{tabular}

In the multivariable forward stepwise regression models, the following variables were tested: age, sex, use of medications, presence of diabetes and hypertension, baseline values and follow-up changes $(\Delta)$ in BMI, HOMA-IR, $\mathrm{HbA}_{1 \mathrm{c}}$, left ventricular mass index (LVMI), blood pressure, waist circumference, WHR and body fat percentage, as well as baseline values of particular left ventricular function variables (i.e. strain, SR and Em)

24]. Reduced energy intake and regular exercise training lead to weight loss and reduced insulin resistance. Weight and adipose tissue loss may contribute to improvement in left ventricular function by decreasing volume overload and cardiac output, attenuating cardioinhibitory cytokine activation and inhibiting neurohormonal disturbances involving the sympathetic nervous system, angiotensin II, leptin, adiponectin and hyperinsulinaemia. The intervention may alter cardiac structural changes including myocyte hypertrophy, interstitial fibrosis, sodium retention and disturbances in myocardial metabolism, e.g. AMP-activated protein kinase [9, 10, 25-27]. Finally, repeated exercise stimulates skeletal muscle glucose uptake and metabolism, thereby improving insulin resistance independently of weight reduction [28].

Clinical effects of improved myocardial function

There is experimental and clinical evidence for the salutary effect of weight loss on cardiac morphology and function [26, 29-35]. Irrespective of the means of weight loss (surgical, pharmacological, dietary or physical training) $[8-10,25,33$, 34], small clinical studies have shown left ventricular mass reduction, improvement in left ventricular diastolic function and a minor increase in left ventricular systolic function [7, $10,36]$. The amelioration of cardiovascular disturbances has been related to the amount of weight loss, with other factors potentially contributing to the observed cardiac benefit receiving little or no attention $[9,37]$.

The present study confirms the favourable effect of lifestyle intervention-induced weight loss and insulin resistance reduction on left ventricular systolic and diastolic function. In participants with good adherence to the dietary and exercise intervention programme, moderate weight reduction and metabolic improvement were observed, as well as favourable alterations in left ventricular mass, plasma lipid profile and cardiorespiratory fitness. In the group without significant weight loss, patients whose insulin resistance improved during the intervention also exhibited left ventricular function benefits. Thus, improvements in left ventricular systolic and diastolic function were associated with weight reduction and decline in insulin resistance. The additive beneficial effect of these two components, as shown in patients from the BMI+IR+ group, supports the notion of their complementary contribution.

Our data suggest that concurrent diabetes diminishes the likelihood of left ventricular function restoration in obese individuals. The reason for this is unclear. However, of all obese participants, those with diabetes mellitus had a greater metabolic and haemodynamic burden (e.g. higher baseline insulin, glucose, HOMA-IR and blood pressure), a greater deposition of visceral fat (a source of biologically active compounds influencing heart muscle) and higher $\mathrm{HbA}_{1 \mathrm{c}}$ (suboptimal glycaemic control is associated with cardiac dysfunction in diabetes). Effective hypoglycaemic treatment might be an important part of successful lifestyle modification in obese patients with diabetes, a notion possibly supported by the finding that a reduction in $\mathrm{HbA}_{1 \mathrm{c}}$ was independently associated with favourable changes in left ventricular diastolic function.

Left ventricular function improvement was clearly dependent on baseline myocardial performance, being more pronounced in patients with greater left ventricular function abnormalities, consistent with prior observations [7]. However, this finding might not apply to patients with overt impairment of left ventricular function, who may not demonstrate a proportionate degree of left ventricular recovery.

Response to the intervention

Left ventricular improvement was shown only in patients with a significant decrease in body weight and/or insulin resistance through the intervention programme, both paralleled by increment in exercise capacity. The participants who responded positively to the lifestyle modifications had a different baseline demographic and clinical profile to the non-responders, being younger and more likely to be of 

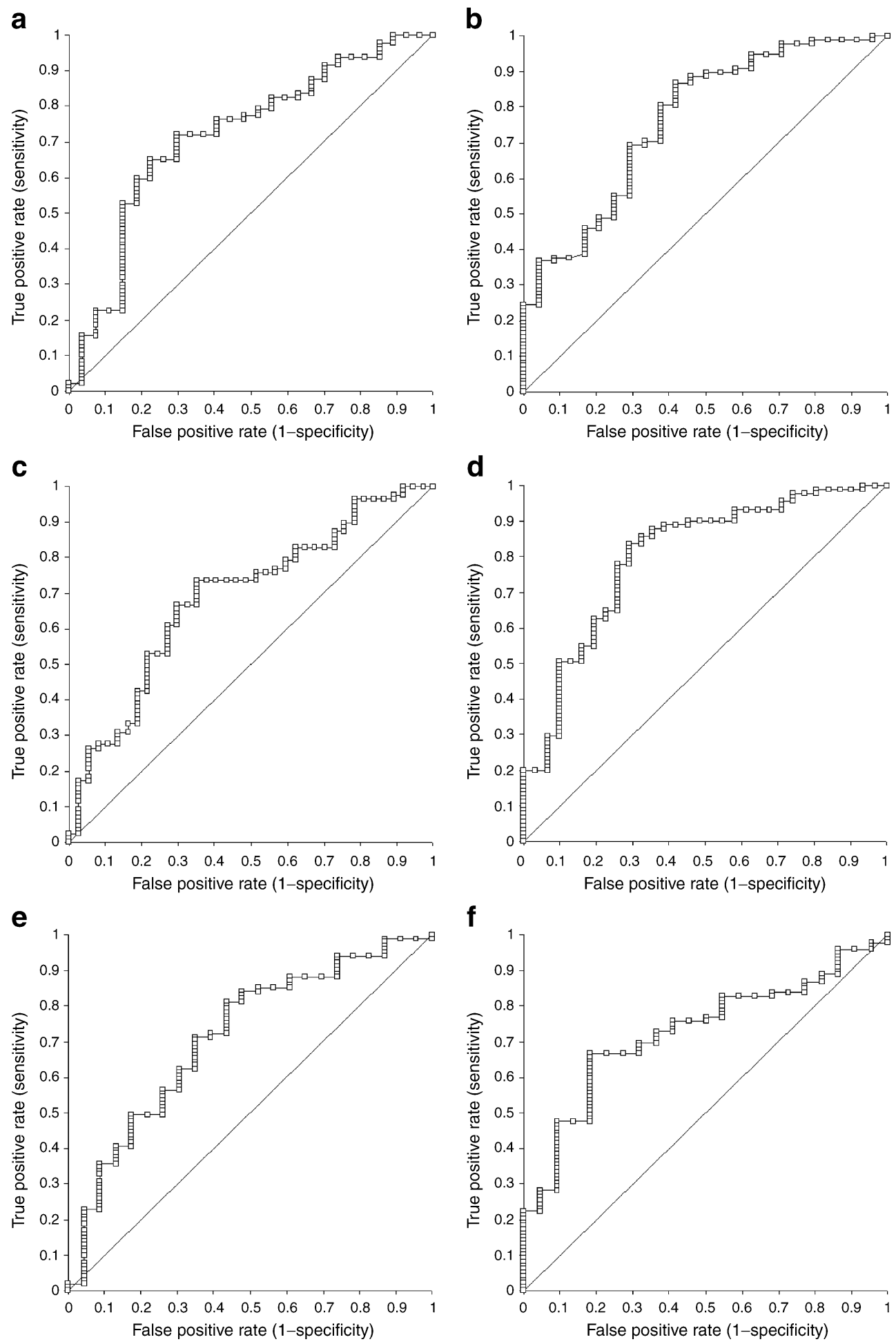

Fig. 1 Receiver operator characteristic curves of the decrease in body weight $(\Delta \mathrm{BMI})(\mathbf{a}, \mathbf{c}, \mathbf{e})$ and insulin resistance $(\Delta \mathrm{HOMA})(\mathbf{b}, \mathbf{d}, \mathbf{f})$ in predicting left ventricular function improvement in obese participants

with good adherence to the intervention. Graphs show curves for strain (a, b), SR (c, d) and Em (e, f). AUC: a $0.71 \pm 0.058$; b $0.76 \pm$ 0.056 ; c $0.69 \pm 0.052 ; \mathbf{d} 0.80 \pm 0.048 ;$ e $0.72 \pm 0.061$; and f $0.73 \pm 0.053$

female sex and to have diabetes, lower visceral fat content and more depressed left ventricular function. These findings may help identify the patients who would be most likely to benefit from this type of lifestyle intervention.

\section{Limitations}

There are several important limitations to our study. First, we have followed myocardial functional indices rather than 

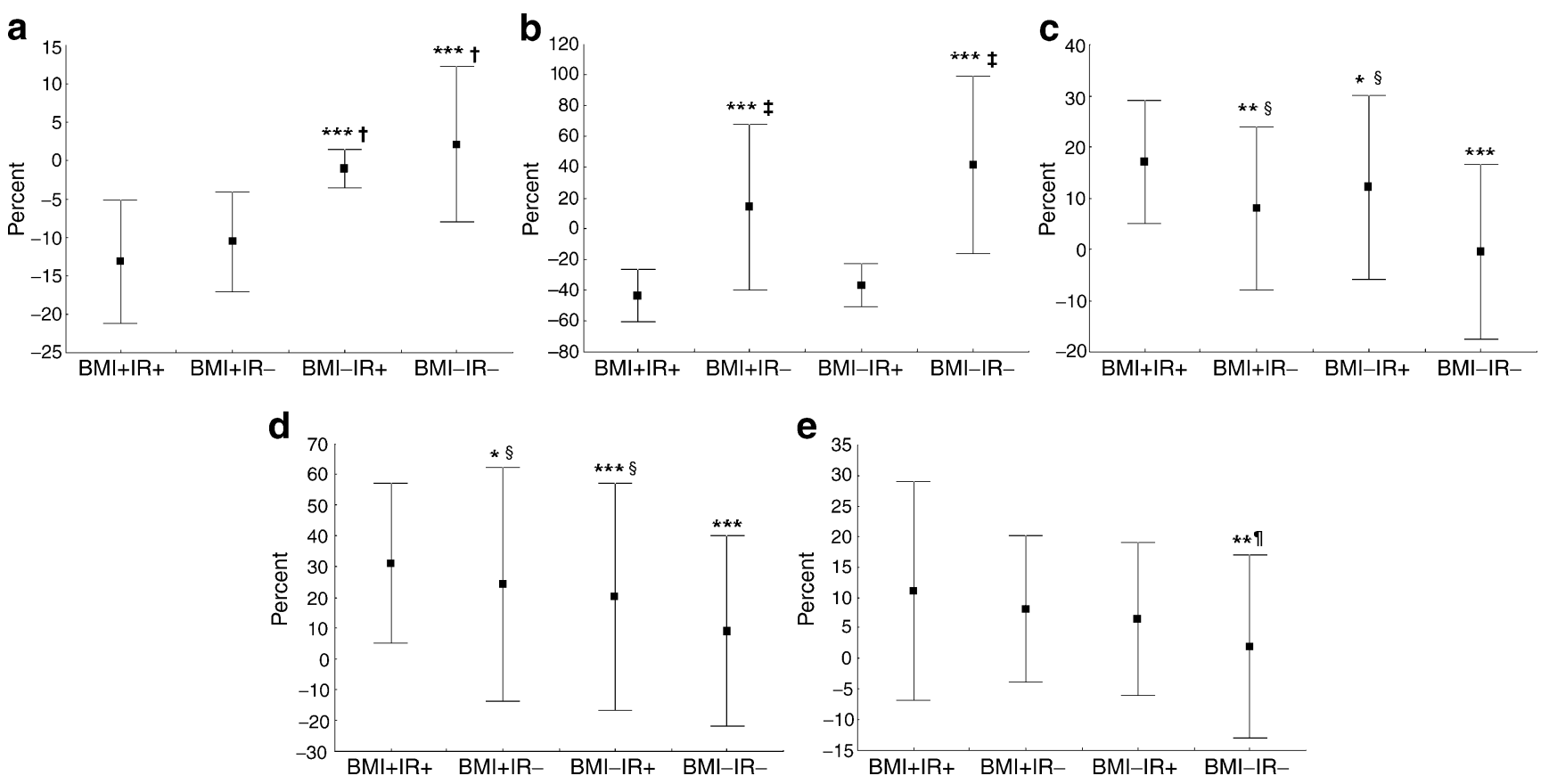

Fig. 2 Changes in BMI (a), HOMA-IR (b), strain (c), Em (d) and $\dot{V} \mathrm{O}_{2}$ (e) in obese patients according to weight reduction and improvement in insulin resistance. Values are follow-up change expressed as per cent of baseline (mean $\pm \mathrm{SD}) .{ }^{*} p<0.05$ vs BMI+ $\mathrm{IR}+; * * p<0.01$ vs $\mathrm{BMI}+\mathrm{IR}+; * * * p<0.001$ vs $\mathrm{BMI}+\mathrm{IR}+; \dagger p<0.001$ vs

BMI+IR-; $\$ p<0.001$ vs BMI-IR+; $\$ p<0.01$ vs BMI-IR-; $\mid p<0.05$ vs $\mathrm{BMI}+\mathrm{IR}-$. $\mathrm{BMI}+$, weight reduction $>5 \%$; $\mathrm{BMI}-$, no weight reduction $>5 \%$; IR + , decrease in insulin resistance $>20 \%$; IR-, no reduction in insulin resistance $>20 \%$

markers of myocardial structure (e.g. calibrated integrated backscatter, cardiac magnetic resonance), which might demonstrate interstitial abnormalities. Second, we anticipated changes in visceral adiposity but do not have imaging

evidence to support this. Third, although no changes to prescribed medications were planned, it is difficult to track all doses over the study interval and this requirement may have not been fulfilled in each case. Fourth, as the goal of

Table 5 Baseline demographic, clinical and echocardiographic characteristics of cardiac function responders to the lifestyle modifications

\begin{tabular}{llll}
\hline Variables & Responders $(n=130)$ & Non-responders $(n=55)$ & $p$ value \\
\hline Age (years) & $42 \pm 13$ & $49 \pm 12$ & 0.001 \\
Sex (\% male) & 24 & 55 & 0.0001 \\
Diabetes mellitus (\%) & 40 & 82 & 0.001 \\
HOMA-IR & $5.1 \pm 4.6$ & $4.4 \pm 3.3$ & 0.31 \\
Waist circumference (cm) & $107 \pm 13$ & $111 \pm 15$ & 0.08 \\
WHR & $0.92 \pm 0.09$ & $0.96 \pm 0.10$ & 0.01 \\
BMI $\left(\mathrm{kg} / \mathrm{m}^{2}\right)$ & $36.3 \pm 5.2$ & $35.0 \pm 6.2$ & 0.17 \\
Body fat $(\%)$ & $39.4 \pm 8.9$ & $37.6 \pm 8.3$ & 0.32 \\
Systolic BP $(\mathrm{mmHg})$ & $128 \pm 15$ & $128 \pm 16$ & 0.96 \\
Diastolic BP $(\mathrm{mmHg})$ & $82 \pm 9$ & $81 \pm 10$ & 0.59 \\
$\dot{V} \mathrm{O}_{2}\left(\mathrm{ml} \mathrm{kg} \mathrm{min}^{-1}\right)$ & $23.3 \pm 8.7$ & $22.9 \pm 8.0$ & 0.79 \\
$\mathrm{LV}$ mass index $\left(\mathrm{g} / \mathrm{m}^{2.7}\right)$ & $50.0 \pm 11.5$ & $47.2 \pm 11.0$ & 0.23 \\
Em $(\mathrm{cm} / \mathrm{s})$ & $6.4 \pm 2.3$ & $5.9 \pm 2.1$ & 0.10 \\
Strain $(\%)$ & $18.3 \pm 2.7$ & $20.9 \pm 3.0$ & 0.0001 \\
SR $(1 / \mathrm{s})$ & $1.45 \pm 0.28$ & $1.60 \pm 0.37$ & 0.002 \\
\hline
\end{tabular}

Unless otherwise indicated, values are mean $\pm \mathrm{SD}$

Non-responders were participants without weight reduction $>5 \%$ and/or insulin resistance decrease $>20 \%$, and without improvement in strain Responders were participants with weight reduction $>5 \%$ and/or insulin resistance decrease $>20 \%$, and improvement in strain 
the study was to understand the links between weight loss and cardiac function, we used a no-weight-loss group as a frame of reference. Unfortunately, weight loss was lower in patients with diabetes than in those without, and the lack of a formal control group limits the ability to exclude confounding due to other changes (e.g. increased muscle mass), although given the lack of change in body composition, we believe this to be improbable. Finally, our study sample was relatively young, predominantly female and white, which limits extrapolation of our findings to groups with different age, ethnic and sex profiles.

\section{Conclusions}

Myocardial function may improve after reduction in body weight and insulin resistance following lifestyle modification in patients with obesity. For these changes to occur, however, programme adherence is required. The cardiac benefit of intentional weight reduction in early-stage disease contrasts with the 'obesity paradox' of late-stage heart failure, where obesity appears to favourably affect prognosis.

Acknowledgements This study was supported in part by a Centres of Clinical Research Excellence award (455832), National Health and Medical Research Council, Canberra, Australia.

Duality of interest The authors declare that there is no duality of interest associated with this manuscript.

\section{References}

1. Murphy NF, MacIntyre K, Stewart S, Hart CL, Hole D, McMurray JJ (2006) Long-term cardiovascular consequences of obesity: 20-year follow-up of more than 15000 middle-aged men and women (the Renfrew-Paisley study). Eur Heart J 27:96-106

2. Centers for Disease Control and Prevention, National Center for Health Statistics (2008) Prevalence of overweight and obesity among adults: United States, 2003-2004. Available from www. cdc.gov/nchs/products/pubs/pubd/hestats/overweight/overwght_ adult_03.htm, accessed 25 March 2008

3. Kenchaiah S, Evans JC, Levy D et al (2002) Obesity and the risk of heart failure. N Engl J Med 347:305-313

4. Di Bello V, Santini F, Di Cori A et al (2006) Relationship between preclinical abnormalities of global and regional left ventricular function and insulin resistance in severe obesity. Int J Obes (Lond) 30:948-956

5. Wong CY, O’Moore-Sullivan T, Leano R, Byrne N, Beller E, Marwick TH (2004) Alterations of left ventricular myocardial characteristics associated with obesity. Circulation 110:3081-3087

6. Ingelsson E, Sundstrom J, Arnlov J, Zethelius B, Lind L (2005) Insulin resistance and risk of congestive heart failure. JAMA 294:334-341

7. Alpert MA, Lambert CR, Panayiotou H et al (1995) Relation of duration of morbid obesity to left ventricular mass, systolic function, and diastolic filling, and effect of weight loss. Am J Cardiol 76:1194-1197
8. Alpert MA, Lambert CR, Terry BE et al (1995) Effect of weight loss on left ventricular diastolic filling in morbid obesity. Am J Cardiol 76:1198-1201

9. Ikonomidis I, Mazarakis A, Papadopoulos C et al (2007) Weight loss after bariatric surgery improves aortic elastic properties and left ventricular function in individuals with morbid obesity: a 3year follow-up study. J Hypertens 25:439-447

10. Alpert MA, Terry BE, Mulekar M et al (1997) Cardiac morphology and left ventricular function in normotensive morbidly obese patients with and without congestive heart failure, and effect of weight loss. Am J Cardiol 80:736-740

11. Look AHEAD Research Group (2007) Reduction in weight and cardiovascular disease risk factors in individuals with type 2 diabetes: one-year results of the Look AHEAD trial. Diabetes Care 30:1374-1383

12. Pi-Sunyer FX, Aronne LJ, Heshmati HM, Devin J, Rosenstock J, RIO-North America Study Group (2006) Effect of rimonabant, a cannabinoid-1 receptor blocker, on weight and cardiometabolic risk factors in overweight or obese patients. JAMA 295:761-775

13. Kosmala W, Wong C, Kuliczkowska J, Leano R, PrzewlockaKosmala M, Marwick TH (2008) Use of body weight and insulin resistance to select obese patients for echocardiographic assessment of subclinical left ventricular dysfunction. Am J Cardiol 101: 1334-1340

14. American Diabetes Association (2008) Diagnosis and classification of diabetes mellitus. Diabetes Care 31(Suppl. 1):S55-S60

15. Alberti KG, Zimmet P, Shaw J (2006) Metabolic syndrome-a new world-wide definition. Diabet Med 23:469-480

16. Anderson KM, Wilson PWF, Odell PM, Kannel WB (1991) An updated coronary risk profile. Circulation 83:356-362

17. Pate RR, Pratt M, Blair SN, Haskell WL, Macera CA, Bouchard C (1995) Physical activity and public health. JAMA 273:402-407

18. Jakicic JM (2003) Exercise in the treatment of obesity. Endocrinol Metab Clin North Am 32:967-980

19. Yokoyama H, Emoto M, Fujiwara S et al (2003) Quantitative insulin sensitivity check index and the reciprocal index of homeostasis model assessment in normal range weight and moderately obese type 2 diabetic patients. Diabetes Care 26:2426-2432

20. Lang RM, Bierig M, Devereux RB, Chamber Quantification Writing Group, American Society of Echocardiography's Guidelines and Standards Committee, European Association of Echocardiography et al (2005) Recommendations for chamber quantification: a report from the American Society of Echocardiography's Guidelines and Standards Committee and the Chamber Quantification Writing Group, developed in conjunction with the European Association of Echocardiography. J Am Soc Echocardiogr 18:1440-1463

21. De Simone G, Devereux RB, Roman MJ, Alderman MH, Laragh JH (1994) Relation of obesity and gender to left ventricular hypertrophy in normotensive and hypertensive adults. Hypertension 23:600-606

22. Ommen SR, Nishimura RA, Appleton CP et al (2000) Clinical utility of Doppler echocardiography and tissue Doppler imaging in the estimation of left ventricular filling pressures: a comparative simultaneous Doppler-catheterization study. Circulation 102:1788-1794

23. Hanley JA, McNeil BJ (1983) A method of comparing the areas under receiver operating characteristic curves derived from the same cases. Radiology 148:839-843

24. Peterson LR, Herrero P, Schechtman KB et al (2004) Effect of obesity and insulin resistance on myocardial substrate metabolism and efficiency in young women. Circulation 109:2191-2196

25. Wong CY, Byrne NM, O’Moore-Sullivan T, Hills AP, Prins JB, Marwick TH (2006) Effect of weight loss due to lifestyle intervention on subclinical cardiovascular dysfunction in obesity (body mass index $>30 \mathrm{~kg} / \mathrm{m}^{2}$ ). Am J Cardiol 98:1593-1598

26. Verreth W, de Keyzer D, Pelat M et al (2004) Weight lossassociated induction of peroxisome proliferator-activated receptor- 
$\alpha$ and peroxisome proliferator-activated receptor- $\gamma$ correlate with reduced atherosclerosis and improved cardiovascular function in obese insulin-resistant mice. Circulation 110:3259-3269

27. Shinmura K, Tamaki K, Saito K, Nakano Y, Tobe T, Bolli R (2007) Cardioprotective effects of short-term caloric restriction are mediated by adiponectin via activation of AMP-activated protein kinase. Circulation 116:2809-2817

28. Hawley JA, Lessard SJ (2008) Exercise training-induced improvements in insulin action. Acta Physiol 192:127-135

29. Freeman GL, Harris MM, Ghidoni JJ, Page A, Cantu TL, Young E (1994) Analysis of myocardial response to significant weight loss in obese rats. Am J Clin Nutr 59:566-571

30. Riordan MM, Weiss EP, Meyer TE et al (2008) The effects of caloric restriction- and exercise-induced weight loss on left ventricular diastolic function. Am J Physiol Heart Circ Physiol 294:H1174-H1182

31. Karason K, Wallentin I, Larsson B, Sjöström L (1998) Effects of obesity and weight loss on cardiac function and valvular performance. Obes Res 6:422-429
32. Ippisch HM, Inge TH, Daniels SR et al (2008) Reversibility of cardiac abnormalities in morbidly obese adolescents. J Am Coll Cardiol 51:1342-1348

33. Dayi SU, Kasikcioglu H, Uslu N et al (2006) Influence of weight loss on myocardial performance index. Heart Vessels 21:84-88

34. Willens HJ, Chakko SC, Byers P et al (2005) Effect of weight loss after gastric bypass on right and left ventricular function assessed by tissue Doppler imaging. Am J Cardiol 95:1521-1524

35. Karason K, Wallentin I, Larsson B, Sjostrom L (1997) Effect of obesity and weight loss on left ventricular mass and relative wall thickness: survey and intervention study. BMJ 315:912-916

36. Gondoni LA, Titon AM, Silvestri G et al (2007) Short term effects of physical exercise and low calorie diet on left ventricular function in obese subjects: a tissue Doppler study. Nutr Metab Cardiovasc Dis 17:358-364

37. Ziccardi P, Nappo F, Giugliano G et al (2002) Reduction of inflammatory cytokine concentrations and improvement of endothelial functions in obese women after weight loss over one year. Circulation 105:804-809 